\title{
Correction of Large-Spread-Angle Stray Light for Measurements of Longitudinal Magnetic Signals
}

\author{
Jiangtao $\mathrm{Su}^{1} \dagger$ and Hongqi Zhang ${ }^{1}$ \\ ${ }^{1}$ National Astronomical Observatories, Chinese Academy of Sciences, Beijing 100080, China \\ email: sjt@sun10.bao.ac.cn
}

\begin{abstract}
To examine the stray light in magnetograph observations, we determined the point spread function of the Video Vector Magnetograph mounted on the Solar Magnetic Field Telescope (SMFT) installed at the Huairou Solar Observing Station (HSOS). Then we obtained the curve on large-spread-angle (LSA) stray light intensity as a function of distance from disk center. A new way to correct LSA stray light is proposed.
\end{abstract}

Keywords. Instrumentation, magnetic fields

\section{Determination of LSA Stray Light intensity}

The observed Stokes I is

$$
I^{o b s}=a_{n o n} I+\sum_{j} c_{j} \Phi\left(r, b_{j}\right)+c_{l} \Phi_{l}(r, b),
$$

where the contribution functions $\Phi\left(r, b_{j}\right)$ and $\Phi_{l}(r, b)$ are numerically calculated by using the formula given by Martinez Pillet (1992). All the coefficients of this linear combination are to be obtained. To the end, we make a linear-square fit to the aureole data. The data we use in the present study were obtained with the HOSO Video Vector Magnetograph on 31 May 2004. We adopted the research method of Chae, et al. (1998) how to select the data to be fitted. Figure 1 shows the intensity profile across the limb and the result of model fitting. In the process of fitting, two Gaussians were used and the corresponding width parameters are $b_{1}=2.5^{\prime \prime}$ and $b_{2}=12.5^{\prime \prime}$. The width parameter of Lorentzian is $b=100^{\prime \prime}$. The LSA stray light intensity integral is presented as a dot-dashed curve. Since it slowly increases with distance from the disk center, the same integral might be used in observations from different days. In the next section, we will use the curve (for convenience, named correcting curve) of it vs. the distance to correct LSA stray light.

\section{Correction of LSG Stray Light}

In routine observations, what we should do first is to make telescope point to the Sun limb to measure background noise signal (BNS) coming from stray light and instruments, then subtract them from polarized light intensity. These BNS are called as Black Level (BL). Since we subtract off a constant background, this method should be suitable only to correct LSA stray light. However, it is clear that the stray light intensity near an active region (AR) is not equal to that of Sun limb. Now, based on the results of section 1 , we propose a new method to correct a constant BNS, which is a LSA stray light in

$\dagger$ Present address: 20A Datun Road, Chaoyang District, Beijing, China. 


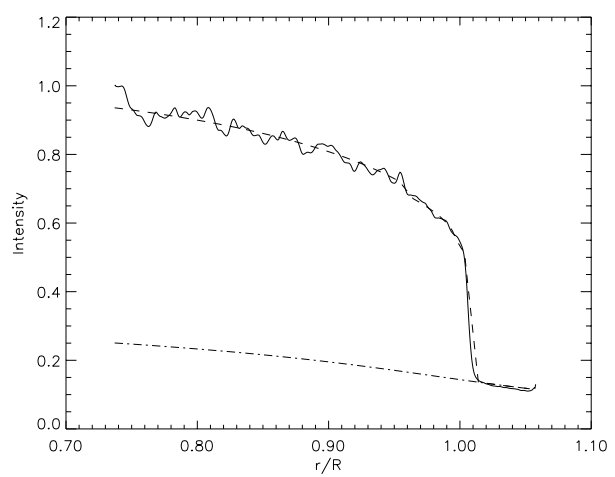

Figure 1. The observed intensity profile across the Sun limb (solid line). The model fit is drawn as dashed line, and the LSA stray light integral is presented as a dot-dashed curve.

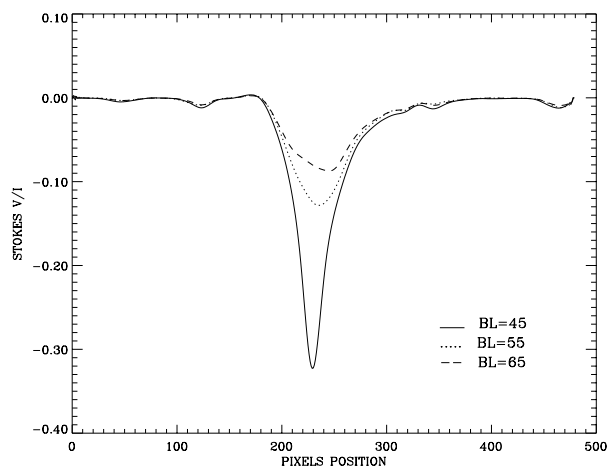

Figure 2. Stokes V/I observed with BLs of 45, 55, 65. vs. pixel location.

the instrument. If BL of Sum limb symbol as $L_{r=1}$, we can get a formula for BL near an $\mathrm{AR}$ on the solar disk by the correcting curve in Figure 1, as

$$
L_{r}=L_{r=1}\left(I_{r} / I_{r=1}\right)-\left(I_{r}-I_{r=1}\right) L_{z e r o} / I_{r=1},
$$

where $L_{z e r o}=110$. We apply it to the AR 10218 to obtain the 'real' BNS (its distance from disk center being $r \sim 0.5$ ), which was observed with different BLs from 35 to 80, in step of 5 . The BL of Sun limb is 65 known from the real time monitor. The rough ratio of LSA stray light intensity of $r=1$ to that of $r=1$ is 1.93 according to the correcting curve. So BL of $r=0.5$ is $\sim 43$ by equation (2). Figure 2 shows the distributions of Stokes $\mathrm{V} / \mathrm{I}$ (observed with BL of $45,55,65$ ) VS. pixel location. We think Stokes V/I with BL of 45 is closer to the 'real' V/I than the others. However, some artificial signal may be added into it. This mainly ascribes to the correcting data for AR 10218 observations obtained in different days.

\section{Acknowledgements}

The authors gratefully thank Yuanyong Deng and Shudong Bao for constructive discussion. We also thank Guoping Wang and Hongwei Qi for their laborious observations.

\section{References}

Chae, J., Yun, H. S., Sakurai, T., and Ichimoto, K. 1998, Solar Phys. 183, 229

Martinez Pillet, V. 1992, Solar Phys. 140, 207. 